\title{
Validity and reliability of the Persian version of the Dutch musculoskeletal questionnaire
}

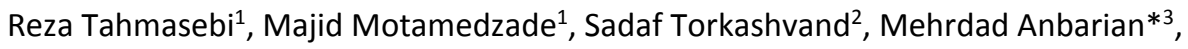 \\ Meysam Olfatifar ${ }^{4}$, Fatemeh Sarvi ${ }^{5}$, Maryam Farhadian ${ }^{6}$ \\ Received: 8 Dec 2017 \\ Published: 4 Apr 2019
}

\begin{abstract}
Background: Dutch Musculoskeletal Questionnaire (DMQ) is used to examine physical and environmental risk factors and to identify high-risk groups for exposure to the risk factors of musculoskeletal disorders at workplace. The aim of this study was to develop the Persian version of the DMQ and to assess its psychometric properties.

Methods: This study was conducted on 212 employees of Gas Transmission Company. Using a standard forward-backward translation procedure, the English version of the questionnaire was translated into Persian and culturally adapted. Content validity was established by 10 expert opinions. The intraclass correlation coefficient (ICC) was used for the statistical analysis of reproducibility. Kuder-Richardson-20 was used for internal consistency and confirmatory factor analysis for structural validity.

Results: This study revealed high internal consistency and good test-retest reliability for the Persian version of the questionnaire. The means of the content validity ratio (CVR) and content validity index (CVI) for the total 35 items of the questionnaire were 0.96 and 0.91 , respectively. The internal consistency of the factors was satisfactory, ranging from 0.83 to 0.88 . Confirmatory factor analysis confirmed the existence of 7 factors in the questionnaire and an acceptable fit for the Persian version of the DMQ.

Conclusion: The Persian version of the DMQ has acceptable psychometric properties. Thus, it can be used as a valid instrument to assess workload and to identify high-risk groups of musculoskeletal disorders at workplace.
\end{abstract}

Keywords: Ergonomics, Dutch musculoskeletal questionnaire, Validity, Reliability

Conflicts of Interest: None declared

Funding: Vice-chancellor for Research and Technology, Hamadan University of Medical Sciences

\section{*This work has been published under CC BY-NC-SA 1.0 license.}

Copyright $\odot$ Iran University of Medical Sciences

Cite this article as: Tahmasebi R, Motamedzade M, Torkashvand S, Anbarian M, Olfatifar M, Sarvi F, Farhadian M. Validity and reliability of the Persian version of the Dutch musculoskeletal questionnaire. Med J Islam Repub Iran. 2019 (4 Apr);33:27. https://doi.org/10.47176/mjiri.33.27

\section{Introduction}

Nowadays, the incidence of musculoskeletal disorders is high among workers. These disorders, in addition to their effect on workers' health, impose high costs for organizations (approximately between $0.5 \%$ and $2 \%$ of gross domestic product (GDP) in some countries) $(1,2)$. Therefore, prevention of work-related musculoskeletal disorders

\footnotetext{
Corresponding author: Dr Mehrdad Anbarian, anbarian@basu.ac.ir

1. Department of Ergonomics, School of Public Health, Hamadan University of Medical Sciences, Hamadan, Iran

2. School of Public Health and Safety, Shahid Beheshti University of Medical Sciences, Tehran, Iran

3. Department of Sport Biomechanics, Faculty of Sport Sciences, Bu-Ali Sina University, Hamadan, Iran

4. Student Research Committee, Hamadan University of Medical Sciences, Hamadan, Iran

5. Department of Biostatistics, School of Health, Hamadan University of Medical Sciences, Hamadan, Iran

6. Department of Biostatistics, Modeling of Noncommunicable Diseases Research Center, School of Public Health, Hamadan University of Medical Sciences, Hamadan, Iran
}

(WMSDs) has been considered as a national priority (3). These disorders have been found to be a serious problem at workplace and can lead to reduced physical ability, disease, and early retirement of workers (4-7). Furthermore, it is the main risk factor of work loss days and dissatisfaction among worker s and one of the important causes of

$\uparrow$ What is "already known" in this topic:

Dutch Musculoskeletal Questionnaire (DMQ) is a valid and reliable tool for measuring self-report musculoskeletal workload, dangerous conditions, and related symptoms in different working groups. This questionnaire allows making a quick but comprehensive survey of the work environment.

\section{$\rightarrow$ What this article adds:}

This study investigated the psychometric properties of the Persian version of DMQ and showed that this version can be considered as an acceptable tool in future studies. This questionnaire can be used to rank different worker groups for exposure to musculoskeletal disorders risk factors. 
disability and absence in many jobs $(8,9)$.

The incidence of work-related musculoskeletal disorders is intensified by various factors present in the workplace, including inappropriate postures, mechanical pressure in certain parts of the body, repetitive movements, intense activity, and static work (10). Several studies have determined the relationship between work-related diseases and workplace physical risk factors $(11,12)$. Many workers in industrial environments are exposed to work-related discomfort and pain. Occupational risk factors are the most important threatening causes to the health of workers (13). Work-related musculoskeletal disorders reduce performance and health of workers $(14,15)$. Studies have shown that workers with low performance can have substantial economic and social consequences $(16,17)$. However, the main cause of musculoskeletal disorders is the delayed identification of exposure to the risk factors for these disorders at workplace $(18,19)$.

To prevent work-related musculoskeletal disorder, it is important to identify physical and environmental risk factors of WMSDs at workplace to categorize different working groups in workload to design work-rest cycles. The Dutch Musculoskeletal Questionnaire (DMQ) is a useful tool for assessing risk factors of musculoskeletal disorders in 7 different dimensions at workplace and identifying high-risk groups in terms of exposure to these risk factors. The first objective of this study was to describe the process of cross-cultural adaptation of the DMQ to Persian and to verify the psychometric properties of the questionnaire (ie, reproducibility, structural validity, internal consistency). The second objective was to assess the risk factors of WMSDs and identify high-risk groups in Iran Gas Transmission Company. Based on the objectives of this questionnaire, (identifying working groups in terms of exposure), 3 different working groups were selected for evaluation.

\section{Methods \\ Study population and data collection}

This cross sectional study was conducted on the employees (administrative, operational, welder employees) of the Gas Transmission Company located in Hamadan Province during October 2016 to March 2017. Out of 212 employees in the study, 15 dropped out and analyses were done on 197 participants, of whom 107, 55, and 35 were administrative, operative, and welder staff, respectively. All eligible participants had 2 years of work experience.

Data were collected using office automation. After performing the necessary coordination, the Persian version of the questionnaire was loaded into the company's automation system, and the staff from different parts were asked to complete the questionnaire. In some parts, where the staff did not have access to the automation system (operational staff and welders), department officials were asked to collect data by giving questionnaires to the workers. The participants were ensured that their information would be kept anonymous; the response rate was $92.2 \%$. This study was approved by the Research Center of Iranian Gas Transmission Company, and informed consent was obtained from all eligible participants.

\section{Questionnaire (scoring and translation)}

The DMQ is used to measure self-report musculoskeletal workload, dangerous conditions, and related symptoms. The questions examine musculoskeletal disorders risk factors but do not examine discomfort rate introduced by exposure to risk factors (20). Questionnaire completion does not require any training. This questionnaire allows making a quick but comprehensive survey of the work environment. This questionnaire has 55 questions and 7 dimensions. The answer to all questions is binary (Yes/No). The scope of the questionnaire includes a force applied, dynamic loads, static loads, repetitive loads, ergonomic environment, vibration, and atmospheric conditions. There are also 4 questions about work posture that are not related to any factor and come at the first part of the questionnaire.

\section{Scoring}

The scoring method was based on participants' positive answers (One point was given for positive answers.). The maximum score for each dimension is equal to the number of questions in that dimension. Standardization was needed considering the difference in the number of questions in each dimension and the necessity of comparing dimension scores with each other. Therefore, the scores in each dimension ranged from 0 (minimum) to 10 (maximum).

\section{Translation}

In the first stage of the study, the original version of the questionnaire, was translated into Persian using the standardized forward-backward translation method (21). In this method, first, the original version of the questionnaire was translated into Persian by 2 professional translators, independently. Next, these versions were combined and analyzed. Then, the combined version was again translated into English by an independent translator from the firstlevel translators who had no access to the original version of the questionnaire. Eventually, during the exchange sessions, the translated and original versions were compared and, finally, the Persian version of the DMQ was prepared.

\section{Statistical analysis}

Content validity: Quantitative and qualitative methods were used to determine content validity. Content validity ratio (CVR) was used in the content quality process, and 10 ergonomics and occupational health professionals were asked to check the necessity of each scale based on the 3part spectrum: (1) essential, (2) useful but not necessary, and (3) not necessary (22). Considering the number of experts who had access to questions, the minimum acceptable value was considered at 0.62 to confirm each item in the next step. Content validity index (CVI) was used to evaluate quantitative content validity. Experts were asked to classify questions based on simplicity, relevance, and clarity in the 4-part spectrum $(1=$ not relevant; $2=$ somewhat relevant; $3=$ quite relevant; $4=$ highly relevant classes for relevance). For each item, CVI was calculated by summing up the number of experts giving a rating of either 3 or 4 and dividing it by the total number 
R. Tahmasebi, et al.

of experts. The minimum acceptable value to confirm each item was considered at 0.78 (23).

Reliability: ICC test-retest method was used to verify the repeatability of the final questionnaire. The questionnaires were distributed twice among 30 employees with a fortnight working schedule; then, intercorrelation between them was calculated. Scores were categorized as follow: less than 0.40 (weak), 0.40 to 0.60 (moderate), 0.61 to 0.80 (good), and greater than 0.80 (great) (24). KuderRichardson 20 score was calculated to assess internal consistency of the questionnaire, and a score greater than 0.7 was considered satisfactory (25).

Construct validity: Confirmatory factor analysis (CFA) method was used to evaluate construct validity and adoption dimensional degree between the original and translated versions of the questionnaire. To diagnose and confirm the dimensions of the questionnaire, the following indices were used: $\chi^{2}$ with a degree of freedom $\left(\mathrm{X}^{2} / \mathrm{df}\right)$, comparative fit index (CFI), Tucker-Lewis index (TLI), and root mean square error estimation (RMSEA). Goodness of fit index ranges are as follow: $\mathrm{X}^{2} / \mathrm{df}$ smaller or equal to 3 and CFI greater than 0.9 shows proper fit and greater than 0.95 indicates excellent fitness; TLI index values above 0.9 show a proper fitness and higher than 0.95 suggest an excellent fitness; RMSEA index values less than 0.08 indicate a good model fitness and less than 0.05 suggest excellent fitness $(26,27)$. SPSS version 21 and M-plus version 5 software packages were used for data analysis.

\section{Results}

\section{Demographic information}

In this study, 197 employees of Gas Transmission Company, including 107 administrative staff, 55 operators, and 35 welders were studied. The mean values of age, weight, and height in participants were 38.5 years, $77.4 \mathrm{~kg}$, and $1.75 \mathrm{~m}$, respectively. Also, they had 12.2 years of work experience and worked 7-8 hours per day. Table 1 illustrates the detailed descriptive data of the participants.

\section{Validity and reliability}

Construct and content validity: The presurvey review from a group of experts led to a number of changes in the wording or structure of some items to improve understandability and readability. Some questions were removed due to their low content validity score based on CVR and CVI, and 35 items remained in the questionnaire and reviews and factor analysis were done. The mean of the CVR and CVI for the total 35 items of the questionnaire was 0.96 and 0.91 , respectively. Also, considerable asset value was obtained for all the items. Confirmatory factor analysis was performed to assess construct validity and provide the most appropriate Persian version of the DMQ. Goodness of the fit indices results are as follow: $\mathrm{RMSEA}=0.051, \mathrm{TLI}=0.974, \mathrm{CFI}=0.969,\left(\mathrm{X}^{2}=1234.7\right.$, $\left.\mathrm{df}=499.8, \mathrm{X}^{2} / \mathrm{df}=2.47, \mathrm{p}<0.001\right)$, which showed good fitness of the model. All dimensions of the questionnaire were correlated with each other. The most correlation was related to the applied force dimension with dimensions of
Table 1. Characteristics of the study participants $(\mathrm{N}=197)$

\begin{tabular}{lccc}
\hline Variables & Administrative & Operational & Welding \\
\hline Gender & & & \\
Male & $93(86.9 \%)^{*}$ & $55(100 \%)$ & $35(100 \%)$ \\
Female & $14(13.1 \%)$ & $0(0 \%)$ & $0(0 \%)$ \\
Education level & & & \\
Under diploma & $6(5.6 \%)$ & $7(12.7 \%)$ & $2(5.7 \%)$ \\
Diploma & $20(18.7 \%)$ & $22(40 \%)$ & $9(25.7 \%)$ \\
Associate & $35(32.7 \%)$ & $21(38.2 \%)$ & $14(40 \%)$ \\
Bachelor & $37(34.6 \%)$ & $5(9.1 \%)$ & $10(28.6 \%)$ \\
Master or higher & $9(8.4 \%)$ & $0(0 \%)$ & $0(0 \%)$ \\
Family status & & & \\
Single & $19(17.7 \%)$ & $5(10 \%)$ & $3(8.6 \%)$ \\
Married & $88(82.3 \%)$ & $50(90 \%)$ & $32(91.4 \%)$ \\
\hline$* \mathrm{~N}(\%)$ & & &
\end{tabular}

$* \mathrm{~N}(\%)$

Table 2. The internal consistency of the 7 factors

\begin{tabular}{lcc}
\hline Dimension & No.Items & Kuder-Richardson index \\
\hline Force applied & 5 & 0.887 \\
Dynamic loads & 4 & 0.840 \\
Static loads & 4 & 0.836 \\
Repetitive loads & 5 & 0.871 \\
Ergonomic environment & 6 & 0.887 \\
Vibration & 2 & 0.835 \\
Atmospheric conditions & 5 & 0.878 \\
\hline
\end{tabular}

dynamic loads, static loads, and ergonomic environment, with a correlation ranging from 0.59 to 0.73 (Fig. 2). All items on related factors had a significant load factor. Therefore, an optimal version of the DMQ questionnaire, with 35 items and 7 factors, was obtained from an Iranian population. Fig. 2 represents the results of factor analysis.

Reliability and internal stability: The internal consistency values of the Persian DMQ questionnaire by the KuderRichardson-20 method are presented in Error! Reference source not found., where the good internal consistency of the questionnaire is noted. Reliability results of ICC test were more than 0.84 that indicated a good reliability of questions.

The comparison of the mean scores for each dimension of the questionnaire in the study population showed that "vibration" (2.5) had the lowest mean score and "repetitive loads" (4.9) had the highest mean score among all the studied dimensions. A standardized average score of all studied factors in the 3 groups is depicted in Fig. 1. The percentage of exposures in 3 groups with poor posture, sitting position, standing position, and walking during work is shown in Fig. 3. The final Persian version of the questionnaire has been added in the Appendix 1).

\section{Discussion}

The aim of the present study was to perform a crosscultural adaptation of the DMQ for Persian speakers and to verify the psychometric properties of the translated tool (reproducibility, structural validity, internal consistency) in a sample of Iranian workers. The content validity of the questionnaire showed that cultural, linguistic, and geographical differences of populations should not be ignored in evaluations. Moreover, standardized versions are not expected to have very similar results based on the language, culture, and customs of different nations $(28,29)$. In the final Persian version of the questionnaire, which included 35 items, the content validity of the whole dimensions and each item was high, indicating that the Per- 


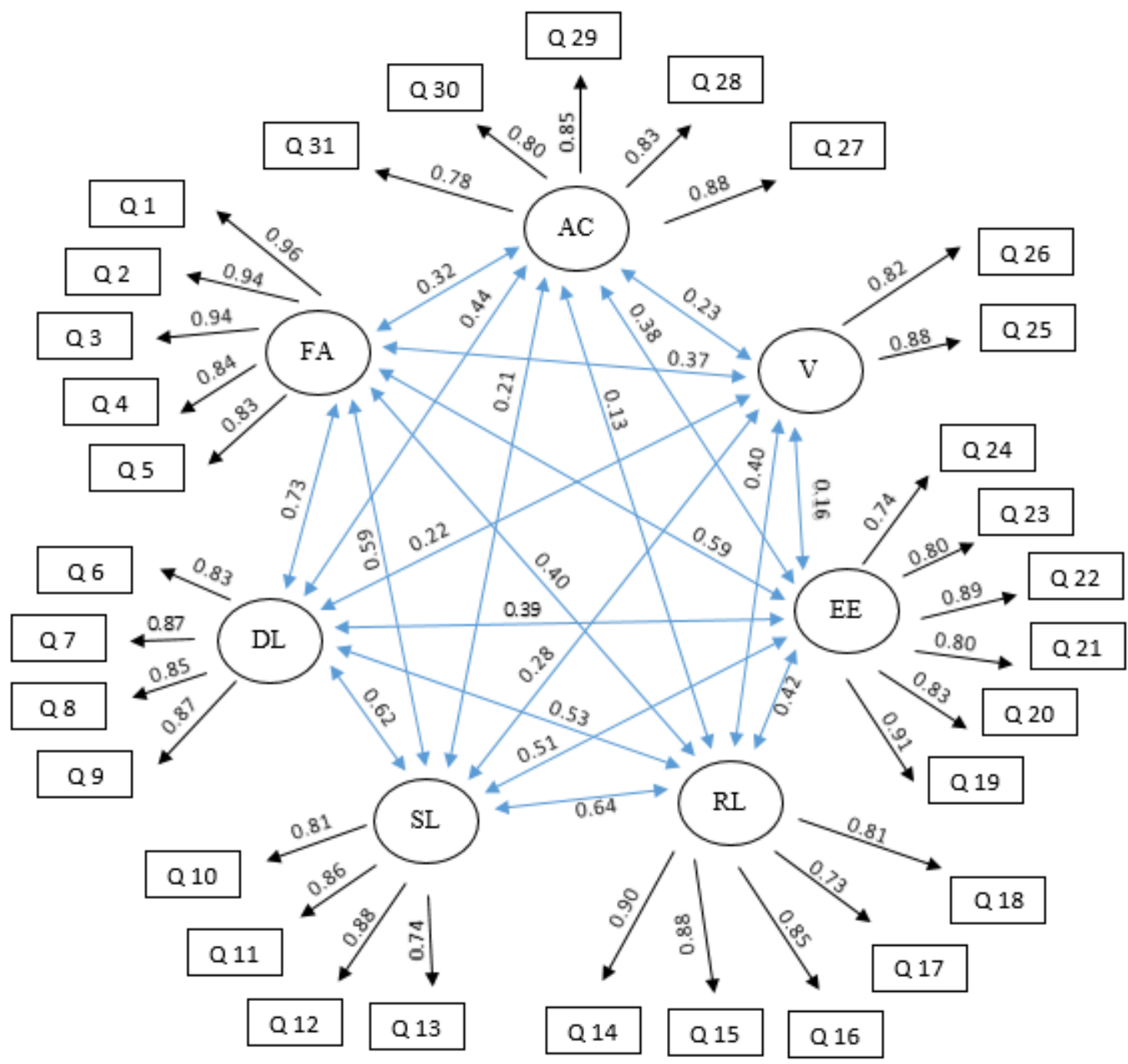

Fig. 2. Diagram showing factor loadings and correlation coefficients estimated by confirmatory factor analysis of the Dutch Musculoskeletal Questionnaire. Note: FA (Force applied), DL (Dynamic loads), SL (Static loads), RL (Repetitive loads), EE (Ergonomic environment), V (Vibration), AC (Atmospheric conditions).

sian version of the DMQ has a good translation and cultural adaptation of the Iranian population.
The internal consistency of the factors was confirmed by measuring Kuder-Richardson-20. Kuder-Richardson-

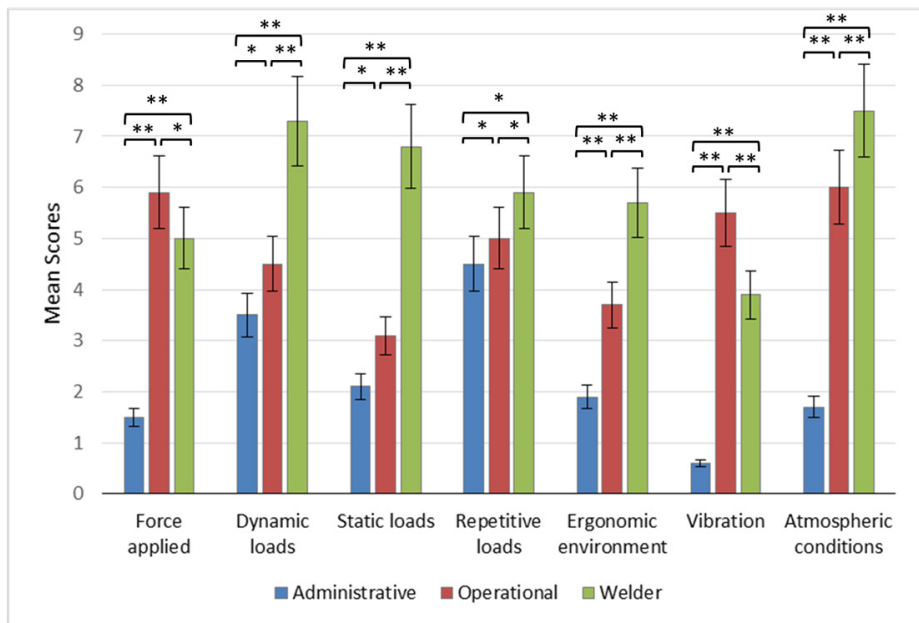

Fig. 1. Standardized mean scores of self-reported exposure summarized in 7 dimensions in 3 groups of the study 


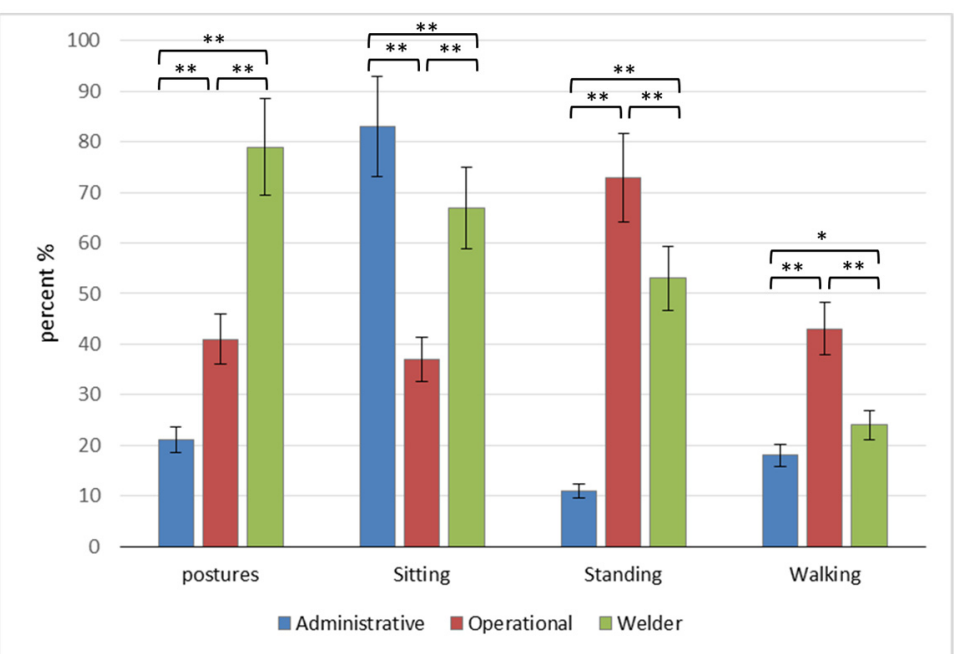

Fig. 3. The mean of self-reported exposure, to uncomfortable postures, sitting, standing and walking in three groups of study. Significant level was set at $<0.05(*)$ and $<0.001(* *)$.

20 coefficient of the Persian version of the DMQ was greater than 0.83 for all dimensions. The high correlation coefficients between items with a dimension indicated the adequacy of their placement in the dimension and further supported the homogeneity of the dimension $(30,31)$. Also, internal consistency evaluation of items showed that all factors had good internal consistency. ICC scores between 0.84 to 1 related to retest, which was repeated after 2 weeks, indicated appropriate reliability of the questionnaire.

Confirmatory factor analysis was selected for verifying the predefined structure of the questionnaire. The findings indicate that the Persian version of the DMQ has a good structural validity. The result of the CFA supported the multidimensional nature of risk factors for musculoskeletal disorders $(32,33)$ and approved all dimensions of the Dutch version of the questionnaire. Correlation between the dimensions of the questionnaire showed a strong, positive, and significant correlation between all dimensions. Therefore, all aspects of DMQ have a close relationship with each other and a change in one of them will affect the others. Moreover, studies conducted on musculoskeletal disorders also confirmed the correlation between musculoskeletal disorder risk factors $(34,35)$.

Comparison of dimensions mean scores in the studied groups showed that welders had higher scores than 2 other groups in dynamic loads, static loads, repetitive loads, ergonomic environment, and atmospheric conditions, suggesting their highest exposure to the above risk factors compared to other groups. Operational workers had the most exposure to force and vibration compared to other groups due to working with vibrating instruments. Administrative staff gained lower scores in all dimensions compared to other groups. According to the result, the risk factors for musculoskeletal disorders can be classified among study groups and used to prioritize corrective action to prevent musculoskeletal disorders. In this study, welders had priority in performing corrective actions in the work environment, followed by operational and administrative staff in the next order.
Analysis of data collected from the 3 study groups (administrative, operational, and welder staff) showed that different groups with conflicting workloads are identifiable. One of the main features of this questionnaire was identification of high-risk groups. However, this distinction is always relative (comparing groups and dimensions with each other) and it may not be possible to define a criterion for high or low exposure based on this qualitative data.

To prevent the occurrence of WMSDs and identify groups at risk of musculoskeletal disorders, a tool is needed to address musculoskeletal disorders risk factors at workplace (36). Using this tool, high-risk groups are identified and appropriate interventions can be used to reduce the incidence of WMSDs. Recent systematic reviews provide robust evidence of manual handling, bending, and turning of the trunk, whole body vibration, repetitive work, heavy physical work, inadequate postures, and workplace design as risk factors of WMSDs $(12,37,38)$. Accordingly, DMQ questionnaire can be used as an appropriate tool for evaluating the risk of musculoskeletal disorders in ergonomic studies by evaluating 7 different domains.

One of the limitations of this study was small sample size. Since the target population of the present study was limited to a company, it was not possible to examine other worker groups (only 3 groups were examined). Also, one of the advantageous features of this questionnaire is identification of worker groups in terms of exposure to WMSDs risk factors, which is suggested to be used in future studies in different worker groups.

\section{Conclusion}

Based on the psychometric properties of the present analysis, the Persian version of the DMQ is a valid and reliable instrument for assessing risk factors for musculoskeletal disorders at workplace. This questionnaire is a useful tool to investigate the physical and environmental risk factors for musculoskeletal disorders at workplace 
and can be used to rank different worker groups for exposure to musculoskeletal disorders risk factors. Nevertheless, further research needs to be done in this area on a larger scale.

\section{Acknowledgements}

The authors would like to thank the management and staff of the Gas Transmission Company, Hamadan University of Medical Sciences, as well as the Head of HSE Department, Mr. Sadegh Afshoun, and Mr. Mehdi Alizadeh for their contribution.

\section{Funding}

This work has been adapted from an MSc thesis at Hamadan University of Medical Sciences, Vicechancellor for Research and Technology, Hamadan University of Medical Sciences (No.9409245181).

\section{Conflict of Interests}

The authors declare that they have no competing interests.

\section{References}

1. Miranda H, Kaila-Kangas L, Heliövaara M, Leino-Arjas P, Haukka E, Liira J, et al. Musculoskeletal pain at multiple sites and its effects on work ability in a general working population. Occup Environ Med. 2010;67(7):449-55.

2. Hauke A, Flintrop J, Brun E, Rugulies R. The impact of work-related psychosocial stressors on the onset of musculoskeletal disorders in specific body regions: A review and meta-analysis of 54 longitudinal studies. Work Stress. 2011;25(3):243-56.

3. Aghilinejad M, Javad Mousavi SA, Nouri MK, Ahmadi AB. Workrelated musculoskeletal complaints among workers of Iranian aluminum industries. Arch Environ Occup Health. 2012;67(2):98-102.

4. Brandt M, Madeleine P, Ajslev JZN, Jakobsen MD, Samani A, Sundstrup E, et al. Participatory intervention with objectively measured physical risk factors for musculoskeletal disorders in the construction industry: study protocol for a cluster randomized controlled trial. BMC Muscoskel Disord. 2015;16(1):302.

5. Woolf AD, Pfleger B. Burden of major musculoskeletal conditions. Bull World Health Organ. 2003;81(9):646-56.

6. Christensen JR, Faber A, Ekner D, Overgaard K, Holtermann A, Søgaard K. Diet, physical exercise and cognitive behavioral training as a combined workplace based intervention to reduce body weight and increase physical capacity in health care workers-a randomized controlled trial. BMC Public Health. 2011;11(1):671.

7. van Rijn RM, Robroek SJ, Brouwer S, Burdorf A. Influence of poor health on exit from paid employment: a systematic review. Occup Environ Med. 2014;71(4):295-301.

8. Christensen KB, Andersen PK, Smith-Hansen L, Nielsen ML, Kristensen TS. Analyzing sickness absence with statistical models for survival data. scand j work environment \& health. 2007:233-9.

9. Niu S. Ergonomics and occupational safety and health: An ILO perspective. Appl Ergon. 2010;41(6):744-53.

10. Punnett L, Wegman DH. Work-related musculoskeletal disorders: the epidemiologic evidence and the debate. J Electromyogr Kinesiol. 2004;14(1):13-23.

11. Kao SY. Carpal tunnel syndrome as an occupational disease. J Am Board Fam Pract. 2003;16(6):533-42.

12. da Costa BR, Vieira ER. Risk factors for work-related musculoskeletal disorders: a systematic review of recent longitudinal studies. Am J Ind Med. 2010;53(3):285-323.

13. Halim I, Omar AR, Saad N. Ergonomic Assessment to Identify Occupational Risk Factor in Metal Stamping Industry. NAME; 2005.

14. Motamedzade M, Ashuri MR, Golmohammadi R, Mahjub H. Comparison of ergonomic risk assessment outputs from rapid entire body assessment and quick exposure check in an engine oil company. J Res Health Sci. 2011;11(1):26-32.
15. Dormohammadi A, Amjad Sardrudi H, Motamedzade M Dormohammadi R, Musavi S. Ergonomics intervention in a tile industry-case of manual material handling. J Res Health Sci. 2012;12(2):109-13.

16. Taieb-Maimon M, Cwikel J, Shapira B, Orenstein I. The effectiveness of a training method using self-modeling webcam photos for reducing musculoskeletal risk among office workers using computers. Appl Ergon. 2012;43(2):376-85.

17. Dul J, Bruder R, Buckle P, Carayon P, Falzon P, Marras WS, et al. A strategy for human factors/ergonomics: developing the discipline and profession. Ergonomics. 2012;55(4):377-95.

18. Ansari N, Sheikh M. Evaluation of work Posture by RULA and REBA: A Case Study. IOSR-JMCE 2014;11(4):18-23.

19. Motamedzade M, Mohseni M, Golmohammadi R, Mahjoob H. Ergonomics intervention in an Iranian television manufacturing industry. Work. 2011;38(3):257-63.

20. Hildebrandt V, Bongers P, Van Dijk F, Kemper H, Dul J. Dutch Musculoskeletal Questionnaire: description and basic qualities. Ergonomics. 2001;44(12):1038-55.

21. Behling O, Law KS. Translating questionnaires and other research instruments: Problems and solutions: Sage; 2000.

22. Lawshe $\mathrm{CH}$. A quantitative approach to content validity. Person Psychol. 1975;28(4):563-75.

23. Polit DF, Beck CT. The content validity index: are you sure you know what's being reported? Critique and recommendations. Res Nurs Health. 2006;29(5):489-97.

24. McGraw KO, Wong SP. Forming inferences about some intraclass correlation coefficients. Psychol. Methods. 1996;1(1):30.

25. Cortina JM. What is coefficient alpha? An examination of theory and applications. J Appl Psychol. 1993;78(1):98.

26. Giles D. Advanced research methods in psychology: Routledge; 2013.

27. Xia P, Li N, Hau KT, Liu C, Lu Y. Quality of life of Chinese urban community residents: a psychometric study of the mainland Chinese version of the WHOQOL-BREF. BMC Med Res Methodol. 2012;12(1):37.

28. Taylor GJ, Bagby RM, Parker JD. The 20-Item Toronto Alexithymia Scale: IV. Reliability and factorial validity in different languages and cultures. J Psychos Res. 2003;55(3):277-83.

29. Beaton DE, Bombardier C, Guillemin F, Ferraz MB. Guidelines for the process of cross-cultural adaptation of self-report measures. Spine. 2000;25(24):3186-91.

30. Andreou E, Alexopoulos EC, Lionis C, Varvogli L, Gnardellis C, Chrousos GP, et al. Perceived stress scale: reliability and validity study in Greece. Int J Environ Res Public Health. 2011;8(8):3287-98.

31. Sanda B, Vistad I, Haakstad LAH, Berntsen S, Sagedal LR, LohneSeiler H, et al. Reliability and concurrent validity of the International Physical Activity Questionnaire short form among pregnant women. BMC Sports Sci Med Rehabil. 2017;9(1):7.

32. Choobineh A, Motamedzade M, Kazemi M, Moghimbeigi A, Pahlavian AH. The impact of ergonomics intervention on psychosocial factors and musculoskeletal symptoms among office workers. Int J Ind Ergon. 2011;41(6):671-6.

33. Morse T, Bruneau H, Dussetschleger J. Musculoskeletal disorders of the neck and shoulder in the dental professions. Work. 2010;35(4):419-29.

34. Kaufman-Cohen Y, Ratzon N. Correlation between risk factors and musculoskeletal disorders among classical musicians. Occup Med. 2011;61(2):90-5.

35. Aghilinejad M, Ehsani AA, Talebi A, Koohpayehzadeh J, Dehghan N. Ergonomic risk factors and musculoskeletal symptoms in surgeons with three types of surgery: Open, laparoscopic, and microsurgery. Med J Islam Repub Iran. 2016;30:467.

36. David G. Ergonomic methods for assessing exposure to risk factors for work-related musculoskeletal disorders. Occup Med. 2005;55(3):190-9.

37. Andersen JH, Haahr JP, Frost P. Risk factors for more severe regional musculoskeletal symptoms: A two-year prospective study of a general working population. Arthritis Rheumatol. 2007;56(4):135564.

38. Mehrparvar AH, Heydari M, Mirmohammadi SJ, Mostaghaci M, Davari MH, Taheri M. Ergonomic intervention, workplace exercises and musculoskeletal complaints: a comparative study. Med J Islam Repub Iran. 2014;28:69. 
R. Tahmasebi, et al.

Appendix 1. Persian version of the Dutch Musculoskeletal Questionnaire

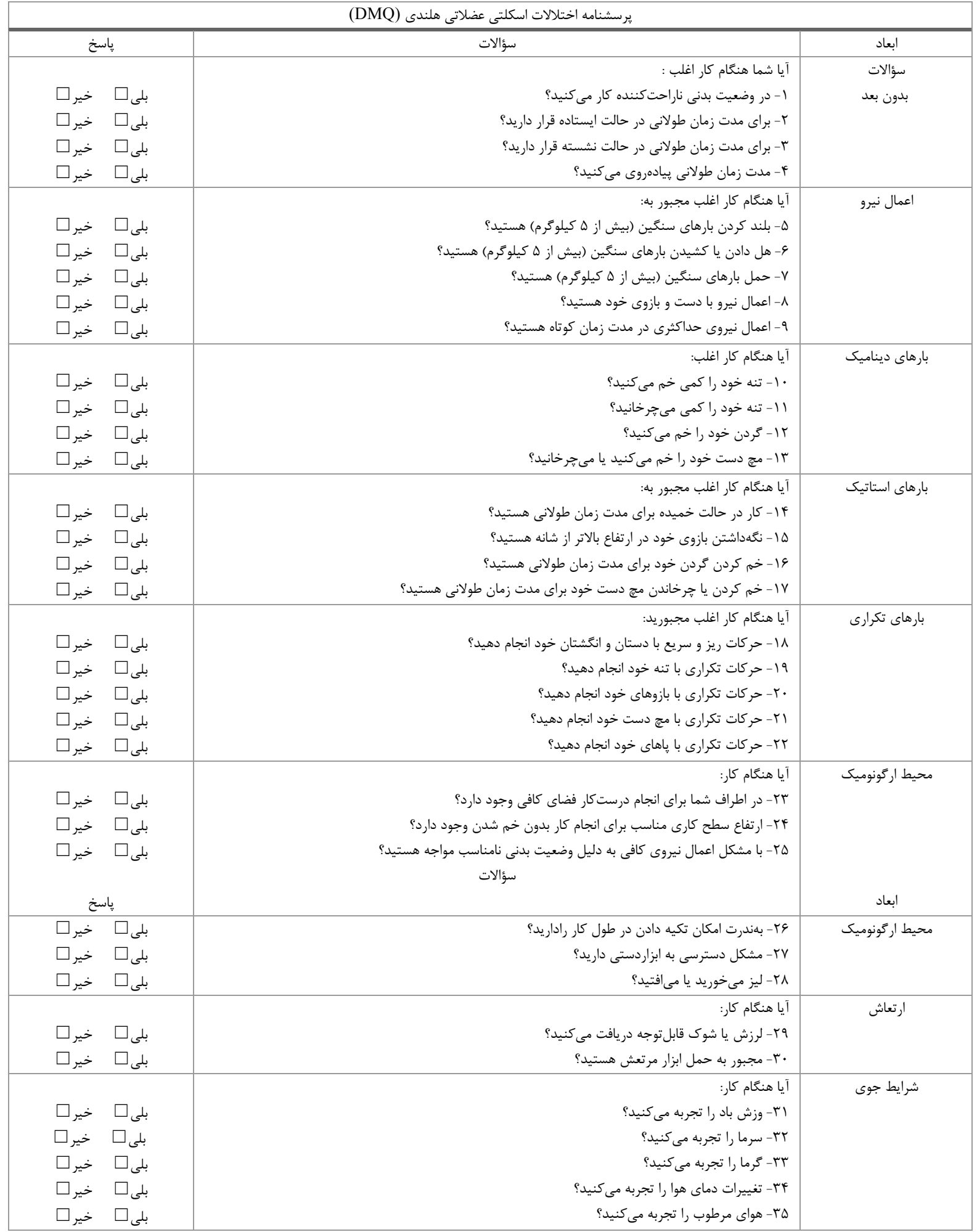

